COMMENTARY

\section{A SCOTTISH RESEARCHER'S RESPONSE}

In ethical debate the questions often matter more than the answers. By raising questions about the anomalies in clinical practice concerning the moral status of the fetus, Boyle et al are contributing to the debate.

There can be no starker reminder of the legal anomaly around fetal/infant rights than the hospital which deals simultaneously with abortions and intensive care of neonates. In the course of my own clinical practice I have recoiled from the horror of late abortions being whisked to the neonatal intensive care unit (NICU), and struggled with the ethical complexities of decision making for infants born at the edge of viability. But many years of detailed researching of the lived experiences of both staff and parents in relation to limitation of treatment, ${ }^{12}$ lead me to the conclusion that to focus discussion on fetal/infant rights is unhelpful. Theoretically the issues may well be conceptualised in this way, but the reality is very different.

It is true that a mother has the right to request termination of her pregnancy at a stage where there is at least some possibility of a viable fetus (although in practice this rarely happens). It is also true that the rights of the fetus may only be realised once it has passed through the birth canal and established itself as a separate individual. A sharp divide at 24 weeks seems illogical, and indeed the whole notion of viability is something of a legal fiction. But in reality things are not as confrontational or as irreconcilable as this paper seems to suggest. Viewed from the perspective of the doctors, nurses, and parents at the coalface of NICU, talk of the child's rights and interests suddenly superseding those of the parents is a distortion; and a picture of arbitrary conflicting reactions on the two sides of this boundary simply does not reflect what happens. Let us look at these two points in more detail.

In clinical situations much more emphasis is placed on the concept of best interests than is ever given to competing rights. This picture was reinforced over and over again in our research. Women voluntarily spent weeks on bed rest to prolong the period of gestation and give the child a better chance of life. Fathers deliberately distanced themselves from the preterm neonate during decisions about withdrawal of treatment to make sure they did not allow personal emotion to prejudice wise judgements. Difficult decisions had to be made but the consensus view of parents was that it was far better that they themselves suffer the agony of losing the child, than that the baby endure undue physical pain. We were left in no doubt that the baby's interests were paramount. For such families the child's interests supersede their own, no matter the gestation.

As for the thinking and practice of the medical team, the picture presented by Boyle et al does not reflect practice as I know it. And my view is based on careful investigation of practice in Scotland and anecdotal evidence from colleagues south of the border. Where the authors have quoted our research, I fear they have rather distorted the results.

Resuscitation and aggressive treatment always carry risks. Survival in a damaged form may not be preferable to death. Judgements about the relative benefits of treatment must therefore be made. Boyle et al state that in practice medical teams usually make treatment withdrawal decisions, even to the point of actively resuscitating infants born on the edge of viability against parental opposition. Clearly in emergency situations it is not always possible dynamically to consult parents about their choices when a preterm delivery is imminent. Sometimes doctors must act first and discuss later. But our research does not support this picture of automatic aggressive treatment at 24 weeks without consideration of the human cost or parental preference-or of a "patriarchal" system. It is not a common scenario in Scotland anyway. Not only is there an increasing tendency for obstetricians to involve the paediatric team at an early stage of discussions, but parents are also key players in the process of decision making. The extent to which they take responsibility for the final choice varies between units and from clinician to clinician, but our findings show that they wish to be given the opportunity to be involved in the discussions, and the majority are satisfied with the extent to which they contribute to the decisions. Great emphasis is placed on the sequencing of events to give parents time, wherever possible, to assimilate the hard facts relating to survival and disability. Tactics such as a staged withdrawal/withholding of extraordinary treatments, providing a sort of "trial of life," showing parents evidence of impairment, offering second opinions, are all adopted to facilitate meaningful involvement of the parents.

It is clear from our data that the medical team and the parents bring different priorities as well as different skills and perspectives to the decision making process. Doctors tend to look first at more technical and prognostic medical factors to assess the chances of survival and the factual consequences of treatments, and then weigh up the implications in terms of pain and future impairment. But for the parents it is the child's welfare that preoccupies them at each stage: suffering and the prospect of a poor quality of life are key factors which influence their opinions. Doctors provide medical expertise, knowledge and experience; parents are uniquely able to give insights into the family's beliefs, values, tolerances and preferences. Together they work towards a shared understanding and conviction as to the most appropriate decision in these circumstances for this child.

Occasionally conflicts do arise between parental and medical judgements, although from our data we know this to be rare. Where it threatens, situations are handled with great sensitivity in most cases. In our reporting, where we have used the term "override" or "overrule" it has been in the context of the overall direction of effort. If one or other side believes the child should be given every chance of life then in order to continue negotiations and assessment of the best interests of the child it is necessary for the child to be kept alive in the interim.

Certainly doctors do not end the lives of children at the whim of parents. The law does not permit arbitrary allowing to die where there is a possibility of meaningful life, and doctors have a duty of care to these patients. But in using the term "overrule" in the way they have, Boyle et al give an impression of doctors unilaterally making life and death decisions regardless of parental wishes. In reality what we described in cases of disagreement was a process of allowing time for the reality of the situation to impinge on the parents' awareness, of gently persuading parents that their perception of the situation does not match with the experience and medical knowledge of the team caring for the baby. Furthermore we also outlined the lengths to which doctors go to minimise the risk of paternalism diminishing the parents' sense of autonomy.

Sometimes the conflict goes the other way and it is parents wanting treatment continued in cases where the medical team feel it to be pointless. Doctors are not obliged in law to give medically futile treatment, but we found that far from enforcing their right not to do so, they rather take time and effort to show the parents evidence of a poor prognosis and persuade them of the implications for the baby of pursuing active measures. Only when there is no resolution in spite of concerted efforts do they turn-very rarely—to the courts. 
Boyle et al refer to an "abrupt change in attitude towards the fetus in utero and the child ex utero at similar gestations" which "may lead to conflict." In their case history they suggest that there is no alternative to full aggressive treatment for a child born at 25 weeks or later, and relate this to the acquisition of a higher moral status. This does not reflect practice north of the border as we have studied it. Here compassion and caution temper the use of technology and I found no evidence of the suggested "conflict".

The authors propose a period of "intermediate parental rights" to cover the period between 24 and 26 weeks, giving parents the right to sanction limitation of treatment. Although I am uneasy about the linking of these rights to specific gestation periods, and I share the opinion of many eminent neonatologists that laws or rules in this area are unworkable and dangerous, I believe that parents do already have the facility to influence and be involved in treatment decisions during this period, just as the mother is before 24 weeks and both parents are after 26 weeks. In reality a practice prevails in Scotland where neonatal staff are in general sensitive to parental wishes and views at every gestation, and in my judgement this seems preferable to a system which accords rights to the parents that conflict with those of the baby.

All treatment carries risks and potential burdens, and choices have to be made. They will be made by fallible people who will not always get it right, but the alternative is to impose a rigid menu of decisions to be applied automatically. The consequences of that are too intolerable to contemplate. Doctors, lawyers, philosophers, and many others have long debated these issues, and the consensus has usually been that we can do no better than to encourage those with the most experience and expertise in these matters to negotiate sensitively in every case with those whose interests are most at stake, and strive to arrive at the best possible solution in these circumstances for this baby and his family at this time.

Issues of "a life not worth living" and just when a disability becomes intolerable are emotive ones and difficult to determine. So much depends on the circumstances, knowledge, and experience of the assessor. In reality we found that the personal opinions of parents as to their own tolerances and attitudes are listened to. It is noteworthy that, of the parents who took part in our study, many referred to their own previous total ignorance of problems in the neonatal period, and as many as $78 \%$ said that their views and priorities had changed as the result of personal experience. It would then surely be ethically dubious for doctors to take parents' initial gut reactions at face value in deciding whether or not to treat babies. There must be some effort made to give them an awareness of the risks of impairment, disability, and death as well as the potential consequences of their choices. Clinical judgements have to be made about just when information and recommendation override parental autonomy.

Of course neonatal intensive care is expensive. The care of damaged infants is a heavy drain on society. Healthcare professionals cannot ignore the issue of limited resources. However in our research it was clear that staff believe, and parents concur, that if wise moral decisions are to be made, these matters cannot be allowed to dictate medical choices at an individual level in the nursery or delivery room. This is a debate which belongs away from the cotside.

These are difficult decisions being made by fallible people, but the present system as we have observed it in Scotland seems preferable to a pursuit of medical treatment without true compassion and practical wisdom.

\section{H McHaffie \\ 12 Mayburn Terrace, Loanhead, Midlothian, UK; hazel@mchaffie.f9.co.uk}

\section{REFERENCES}

1 McHaffie HE, Fowlie PW. Life, Death and Decisions: doctors and nurses reflect on neonatal practice. Cheshire: Hochland and Hochland, 1996.

2 McHaffie HE in association with Fowlie PW, Hume R, Laing IA, Lloyd DJ, Lyon AJ. Crucial Decisions at the Beginning of Life: parents' experiences of treatment withdrawal from infants. Oxford: Radcliffe Medical Press, 2001.

\section{$\mathrm{ECHO}$}

\section{Necropsies have value in medical education}

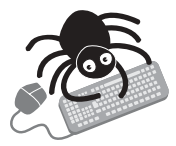

Please visit the Journal of Medical Ethics website [wmw. jmedethics. com] for a link to the full text of this article.
$M$

edical educators in the UK still believe necropsy is a valuable educational resource despite recent negative publicity, curricular revisions and declining use of the procedure.

A "theoretical sample" of teaching staff from Sheffield University Medical School completed a semistructured private interview designed to clarify their personal attitudes towards necropsy. Similar statements were grouped together as themes, of which nine were identified at the completion of data gathering and included: a readiness of the participants to consent to their own necropsy; a high degree of clinical detachment from emotions linked to necropsy; and a willingness to consent to necropsy on a close relative. Some participants raised concern that necropsy objectified the human body, whilst others felt the procedure increased the respect paid to the deceased.

The wide range (and occasionally conflicting nature) of responses received in the study reflects the theoretical sampling undertaken. Interestingly, participants who would not give consent for a close relative to receive necropsy stated that it was due to their religious beliefs rather than any doubts about the educational value of necropsy.

A Journal of Clinical Pathology 2003;56:950-951. 